\title{
El teorema fundamental del cálculo: Escenarios para su comprensión'
}

The fundamental theorem of calculus: Scenarios for understanding O teorema fundamental do cálculo: Cenários para a compreensão

Recibido: mayo de 2013

Aceptado: agosto de 2013
Wilson David Peñaloza Castro²

Sonia Rocío Suárez Cáliz ${ }^{3}$

Solange Roa Fuentes ${ }^{4}$

\section{Resumen}

Este trabajo propone tres escenarios construidos en un software dinámico que buscan relacionar el cálculo diferencial y el integral a través de la construcción comprensiva del teorema fundamental del cálculo. Dichos escenarios fueron construidos tomando como base las cuatro fases que sustentan el uso de herramientas tecnológicas en la resolución de un problema propuestas por Santos y Moreno (2013). Se resalta el acercamiento visual y empírico a través de la construcción de la derivada como la pendiente de la recta tangente a una curva, la construcción de la integral definida como el área bajo la curva en un intervalo cerrado y cómo éstas se relacionan en el teorema fundamental del cálculo.

Palabras clave: Aula; recursos didácticos; recursos informáticos; software dinámico; matemáticas escolares; cálculo; teorema fundamental del cálculo; comprensión.

\begin{abstract}
This paper proposes three scenarios built on a dynamic software seek to relate the differential calculus and integral through comprehensive construction of the fundamental theorem of calculus. These scenarios were constructed based on the four phases supporting the use of technological tools in solving a problem proposed by Santos and Moreno (2013). It highlights the visual and empirical approach through the construction of the derivative as the slope of the tangent to a curve, the construction of the definite integral as the area under the curve on a closed interval and how they relate in Theorem fundamental calculation.
\end{abstract}

Keywords: Classroom, teaching resources, computing resources, dynamic software; school math, calculus, fundamental theorem of calculus; understanding.

\footnotetext{
1 Artículo de Investigación.
}

2 Universidad Industrial de Santander. Colombia. Contacto: david_p_1102@hotmail.com;

3 Universidad Industrial de Santander. Colombia. Contacto: joncia91@hotmail.com;

4 Universidad Industrial de Santander. Colombia. Contacto: sroa@matematicas.uis.edu.co 


\section{Resumo}

Este artigo propõe três cenários construídos em um software dinâmico procuram relacionar o cálculo diferencial e integral, através da construção completa do teorema fundamental do cálculo. Estes cenários foram construídos com base nas quatro fases de apoio à utilização de ferramentas tecnológicas na resolução de um problema proposto por Santos e Moreno (2013). Destaca-se a abordagem empírica visual e por meio da construção do derivado como o declive da tangente a uma curva, a construção do integral definida como a área sob a curva num intervalo fechado e como se relacionam no Teorema cálculo fundamental.

Palavras-chave: sala de aula, recursos pedagógicos, recursos de computação, software dinâmico; matemática escolar, cálculo, o teorema fundamental do cálculo, a compreensão.

\section{Introducción}

Este trabajo busca resaltar los elementos visuales en la construcción comprensiva de conceptos y nociones matemáticas. En particular, buscamos generar escenarios dinámicos que mediante la representación geométrica de la derivada y la integral motiven la comprensión del teorema fundamental del cálculo ya que, como afirma Zimmermann:

Conceptualmente, el papel del pensamiento visual es tan fundamental para el aprendizaje del cálculo que es difícil imaginar un curso exitoso de cálculo que no enfatice los elementos visuales del tema. Esto es especialmente verdadero si el curso tiene la intención de promover un entendimiento conceptual, el cual es ampliamente reconocido como carente en la mayoría de los cursos de cálculo tal como se enseña hoy. La manipulación algebraica ha sido enfatizada en exceso... en este proceso el espíritu del cálculo se ha perdido. (1990, p. 136)

Hacer uso de las diversas herramientas tecnológicas para proponer actividades que puedan ayudar a profundizar y comprender conceptos matemáticos, es una problema latente para la Educación Matemática hoy día. Estas herramientas ofrecen un acercamiento visual que permite una mejor comprensión de los conceptos y nociones matemáticas, como plantea De Guzmán (2006): "las ideas, conceptos y métodos de las matemáticas presentan una gran riqueza de contenidos visuales, representables intuitivamente o geométricamente" (p. 15); aprovechar estas características visuales resulta en la mayoría de los casos de gran utilidad en la construcción comprensiva de conocimiento matemático. En particular la propuesta que presentamos se basa en el diseño de tres escenarios en en el software dinámico GeoGebra.

GeoGebra es una herramienta de trabajo que puede ser de utilidad tanto para el docente como para el alumno. Para el docente, porque le permite elaborar materiales didácticos estáticos como presentaciones, trabajos prácticos, etc. o materiales dinámicos, como demostraciones dinámicas. Al alumno le brinda la posibilidad de visualizar conceptos matemáticos, realizar construcciones libres o dirigidas a fin de resolver problemas y/o explorar e investigar hipótesis (MEN Buenos Aires, 2011, p. 16).

Haciendo uso de GeoGebra presentamos tres escenarios particulares que buscan la construcción comprensiva del teorema fundamental del cálculo; para Schank (1982) citado por López (2011) "la comprensión es un proceso de decodificación de las percepciones a través del cual damos un significado a las frases y situaciones de la vida real. Este proceso se basa principalmente en los conocimientos que se hallan disponibles en nuestra memoria" 
(p. 1); de esta forma queremos que el estudiante se apoye en el escenario propuesto e interactúe con él, de modo que logre un proceso de asimilación de los conceptos abordados, de tal manera que pueda comprender las ideas fundamentales que dan origen al teorema fundamental del cálculo.

\section{Elementos que fundamentan los escenarios propuestos}

El diseño de las actividades que hemos denominado como "escenarios", se basa en actividades específicas que en el contexto de GeoGebra, buscan la construcción comprensiva del Teorema Fundamental del Cálculo a partir de la relación entre la representación gráfica de la derivada (como pendiente de la recta tangente a una curva) y la integral (como el área bajo la curva).

Para esto tomamos como referencia las cuatros fases que proponen Santos y Moreno (2013), que se toman como un marco conceptual para la solución de problemas mediante el uso de herramientas computacionales; a continuación describimos cada fase:

Fase I Problematizar: compresión, sentido y representación del problema. Polya (1945, citado por Santos y Moreno, 2013) reconoce que es importante que los estudiantes comprendan y discutan el sentido del enunciado de un problema, o de una idea matemática. Por ejemplo, algunas preguntas que pueden guiar al proceso de comprensión y representación de los escenarios que proponen incluyen: ¿Qué representa cada punto que deja el rastro de $\mathrm{B}$ ? ¿Qué relación hay entre la función $\mathrm{f} y$ la curva que dibuja el rastro de $B$ ?

Fase II. Exploración visual y empírica. En esta fase se resalta la construcción de un modelo dinámico del problema que permite visualizar y cuantificar el comportamiento de los parámetros asociados en el problema (Santos y Moreno, 2013).

Fase III. Búsqueda de múltiples métodos de solución. La búsqueda y discusión de diversas formas de resolver un problema representan un aspecto fundamental en el proceso de construir una comprensión conceptual de las ideas matemáticas en el desarrollo de competencias en la resolución de problemas (Santos y Moreno, 2013).

Fase IV. Episodio de reflexión. Conviene reflexionar acerca de los procesos matemáticos que se exhiben durante las fases de resolución del problema. En la fase inicial resulta relevante formular y discutir abiertamente una serie de preguntas que ayuden a comprender y encontrar el sentido asociado con el enunciado del problema (Santos y Moreno, 2013).

A continuación describiremos con mayor detalle cómo estás fases se desarrollan en un escenario en particular.

\section{Descripción de los escenarios: aspectos generales}

Los tres escenarios que se construyeron en este trabajo están diseñados de tal manera que permiten que los estudiantes comprendan mediante la representación geométrica de la derivada y la integral la relación que se establece en el teorema fundamental del cálculo. A continuación describimos a grosso modo los dos primeros escenarios, que fundamentalmente busca que los estudiantes comprendan geométricamente la relación entre las gráficas de una función, la gráfica de la función derivada y la gráfica de la función integral con la gráfica de la función original.

Escenario I. En primer lugar se propone la construcción de un escenario que permite ver la relación de la función derivada como la pendiente de la recta tangente en un punto a una curva y la gráfica de dicha curva, se plantean preguntas que guían al estudiante a la apropiación de esta relación.

Escenario II. Se propone la construcción de un escenario geométrico que posibilita la visualización de la integral definida de una función en un intervalo $[a, b]$ y la gráfica de la función original para que el estudiante comprenda la relación que existe entre ellas, al igual que en el anterior escenario se 
presentan preguntas claves para la asimilación de esta relación.

Escenario III. Se presenta una construcción que relaciona las gráficas de la función integral y la función derivada con la gráfica de la función original mediante el teorema fundamental del cálculo y así complementar lo que analíticamente se conoce respecto a que la derivada y la integral son procesos inversos.

Las preguntas que aparecen a continuación parten de la exploración de los estudiantes sobre una construcción dada.

Figura 1: Representación del teorema fundamental del cálculo.

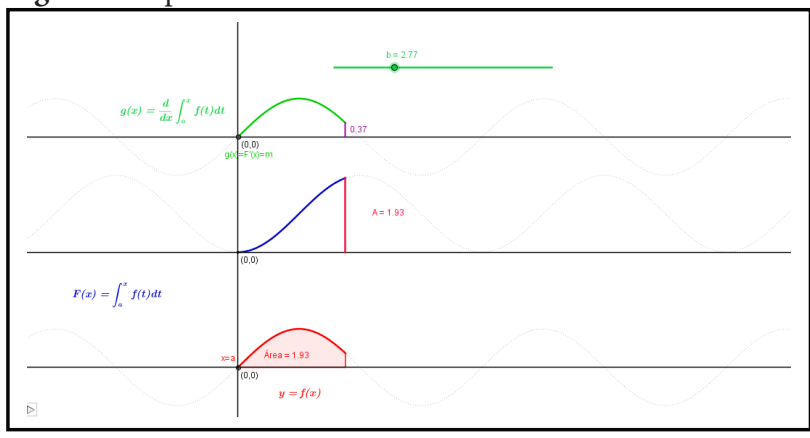

Fuente: Elaboración propia.

A partir de dicha construcción se pide a los estudiantes realizar los siguientes pasos de exploración.

1. Abre el archivo de GeoGebra llamado Teorema Fundamental del cálculo. Mueve el deslizador $b$ mediante el botón que se encuentra en la parte inferior izquierda, a partir de lo que observa en la construcción di qué relación existe entre la función de color rojo con la de color azul.

En esta pregunta se espera que el estudiante tenga en cuenta la relación que se estudió en el escenario II.

2. Dirígete al menú de herramientas y utilizando la opción expone / oculta objeto da clic a la recta morada y a su pendiente, posteriormente da clic al botón de la parte inferior izquierda y con base a lo que ocurre di qué relación existe entre la función de color azul y la de color verde.
En esta pregunta se espera que el estudiante tenga en cuenta la relación que se estudió en el escenario I.

3. ¿Qué relación existe entre la gráfica de color rojo con la de color verde?

Aquí se espera que el estudiante observe que la integral de la derivada de una función es la misma función, comprendiendo lo que plantea el teorema fundamental del cálculo.

\section{Conclusiones y comentarios finales}

El uso de la tecnología en particular el ambiente que ofrece GeoGebra permite a los estudiantes crear representaciones mentales de los conceptos matemáticos, a través de la visualización que hacen al realizar las actividades propuestas por el docente. Proponemos que esto implica una mayor comprensión e interpretación de los conceptos involucrados. La comprensión de las relaciones que se dan entre ejemplos particulares, puede promover la construcción de generalizaciones que sean más intuitivas para los estudiantes. Esto se logra gracias a la problematización de una situación matemática y las potencialidades de representarla en un medio visual.

Durante la presentación de este trabajo, analizaremos los resultados de desarrollar los escenarios propuestos con un grupo de 10 estudiantes universitarios de un programa de Licenciatura en Matemáticas; centrándonos en la potencialidad de desarrollar las fases propuestas por Santos y Moreno (2013).

\section{Referencias}

De Guzmán, M. (1996). El Rincón de la Pizarra. El papel de la visualización. Madrid: Pirámide.

MEN Buenos Aires, (2011). Geometría, Serie para la enseñanza en el modelo 1 a $1 /$ compilado por Paula Podestá. Argentina: Ministerio de Educación de la Nación. 
Santos, M., Moreno, L. (2013). Sobre la construcción de un marco conceptual en la resolución problemas que incorporen el uso de herramientas computacionales. En las tecnologías digitales en la enseñanza de las matemáticas. México: TRILLAS.

López, O., (2011). Comprensión lectora. Chiclayo, Perú. Recuperado de: http:// www.slideshare.net/oscarlopezregalado/ la-comprensin-lectora-7321163

Zimmermann W. (1990) Visual Thinking in Calculus. In Visualization in Teaching and Mathematics (Zimmermann W. \& Cunningham S. Editors), MAA, No.19. 